\title{
FOUNDATIONS FOR WIDE-AREA SYSTEMS
}

\author{
Luca Cardelli (Joint work with Andrew D. Gordon)
}

Microsoft Research, Cambridge, UK

luca@luca.demon.co.uk

The last decades have seen the emergence of the "sea of objects" paradigm for structuring complex distributed systems on workstations and local area networks. In this approach, applications and system services are composed of and communicate among themselves through reliable and transparently accessible object interfaces, leading to the interaction of hundred or thousands of unstructured objects.

This approach has lead to major progress in software composability and reliability. Unfortunately, it is based on a number of assumptions that do not hold on wide-area networks, where access to resources is intrinsically unreliable (because of failure, congestion, disconnected operation, etc.) and not transparent (because of variations in latency and bandwidth, and the presence of firewalls).

In this tutorial we introduce a formalism, the Ambient Calculus, that was devised to match some fundamental characteristics of wide-area networks and systems. Our approach reflects the intuition that, to function satisfactorily on a wide-area network, the sea of objects must be partitioned and made hierarchical, internally mobile, and secure. 